\title{
Fault Locations in Transmission Systems by Evolutionary Algorithms
}

\author{
W. Guerra and N. Kagan \\ ${ }^{1}$ Department of Energy and Automation Engineering \\ Centro de Estudos em Regulação e Energia Elétrica (Enerq-ct) \\ Escola Politécnica - U.S.P, São Paulo University \\ Avenida professor Luciano Gualberto, travessa 3, 158 Bloco A (São Paulo-Brasil) \\ Phone/Fax number:+0055 1130919917, e-mail: willguech@gmail.com, nelsonk@pea.usp.br
}

\begin{abstract}
This paper aims at presenting the development and implementation of an approach to deal with fault location in transmission systems by Evolutionary Algorithms. An Evolutionary Strategy was applied to address an optimization problem, from the generation of a population of individuals that represent possible solutions to the problem, as well as evolutionary operators such as selection, mutation and crossover. Some variants of the basic approach were also considered in such a way to determine the fault location with better performance. A case study, based on the 30 bus IEEE study system, demonstrates the potential of the proposed methodology.
\end{abstract}

\section{Keywords}

Optimization, Fault Location, Evolutionary Algorithms, Evolutionary Strategies.

\section{Introduction}

The Electricity market has been more and more demanding on the levels of power quality, which has lead utilities to improve their network operation procedures by the use of adequate software and hardware, so that a better operation and resulting power quality indices are achieved.

Power quality can be generally divided into two topics, namely quality of service and voltage quality. Quality of service is very much related to reliability and long duration interruption indices, which are commonly caused by power system faults. In such a context, fault location is considered so as to reduce the amount of time in which emergency crews can restore the system. Network faults are random events, which generally lead to actuation of the protection devices, aiming at protecting installations and individuals as well as maintain the power system stability. A rapid power restoration depends on the fault location and the repairing time [1].

Faults in power lines are due to many causes, such as isolation problems, fire near the transmission lines, phase contacts provoked by external agents, amongst others. Most of the transmission line faults involve the contact of one of the phases and the ground, which is phase to ground faults [1]. In such cases, short circuit currents might be reduced due to fault impedances, what makes the network protection more difficult to actually perform as required.

In order to determine an approximate fault location, one needs the characteristic parameters of the power lines, power transformers and other pieces of equipment that compose the given network, as well as the fault type and fault impedance. Another difficult issue relates to the load uncertainty, which makes the problem solution even harder. In most cases, these parameters are not completely known, thus constituting an extra challenge for the fault location problem.

On the other hand, power lines and networks are generally equipped with meters, commonly power and energy meters and some few power quality meters. These latter devices are installed not specifically for fault location but actually to monitor the effects of network faults, such as voltage sags. The low amount of power quality meters in the system, i.e. a few meters are located in the power system buses, makes the fault location problem more complex.

The scientific literature shows some research works that address the solution for fault location problems. A research group has applied artificial neural networks [2], 
by using data from one of the power line terminals, thus reducing the amount of necessary information. Reference [3] uses Bayes Theorem to apply a probabilistic model to the solution of rather complex communication systems. These research works make use of plenty of available data related to the event and loading of the system, thus making their application rather difficult for real conditions, where data information is incomplete.

This paper deals with the application of Evolutionary Algorithms (EAs) [4] for the solution of fault location problems in transmission systems. EAs are widely known as an adequate artificial intelligence tool to solving many electrical engineering problems. The application of such technique is somehow different from Genetic Algorithms (GAs). In EAs, the mutation operator constitutes the main evolutionary operator, which introduces a renewal in population so that new variations are generated on individuals.

However, in a given population of individuals, the main evolutionary operators are used, namely selection, mutation and crossover to attain the optimal or suboptimal solution to the given problem.

The application of such technique to the fault location problem will herein be illustrated by a case study to the 30 bus IEEE network.

\section{Evolutionary Algorithms (EAs)}

The concepts of evolutionary computation can be described, according to [4] by Genetic Algorithms, Evolutionary Programming and Evolutionary Strategies. The former approach has shown fairly promising results from the very beginning. In the same manner as other solution methods inspired in the nature, such as artificial neural networks and fuzzy systems, the evolutionary algorithms had to go through a difficult process of acceptance by the scientific community. During the 1990s however such techniques showed as powerful tools to solve highly complex problems.

The basic structure of an evolutionary algorithm can be given as follows:

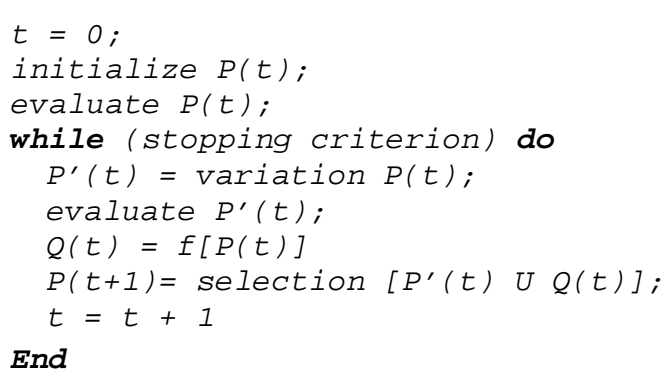

In such algorithm, $P(t)$ denotes a population of $\mu$ individuals in a given generation $t . Q(t)$ represents a set of individuals that can be considered for selection, according to the function $f[$.$] . As illustrative examples,$ $Q(t)$ can be made equal to the $P(t)$ or $Q(t)$ can be made equal to the null set. A new population $P^{\prime}(t)$ of $\lambda$ individuals is then generated through the recombination and mutation operators. New individuals from $P^{\prime}(t)$ are then evaluated by measuring their "distance" to the "target" or optimal solution of the given problem. As a result, a specific fitness function is established for each one individual. A new population is then formed at generation $t+1$, by selecting the most fitted individuals.

As for the fault location problem, a given individual, that represent a possible fault location, will be assessed by how close the calculated values at the meters are to the measured ones. In other words, the fittest individuals are more likely to belong to the following generation.

After a given number of generations, a stopping criterion is to be met, that usually presupposes that a given individual in the population represents the most acceptable solution to the problem. When such criterion is difficult to be previously established, the number of generations is used as an input parameter to determine the end of the process.

\section{Fault Location}

\section{A. Problem Description}

When a permanent fault occurs in transmission lines, emergency crews are called to locate the fault site. This is generally a long process, since one does not know in advance where the fault took place. This implies on high maintenance costs and the reduction of system reliability.

Utilities therefore have looked for fault location systems, which allow for the identification of the point where the fault occur, thus minimizing the emergency crew's displacement time as well as an increase in the system reliability. 


\section{B. Proposed methodology for the fault location problem}

As aforementioned, the methodology makes use of Evolutionary Strategies (ES) [4]. The evolutionary operators will be herein described, by envisaging the fault location problem.

The individual's codification is the first step that relates the real problem to the ES application.

\section{Codification}

Individuals are represented by the variables concerning to the fault location problem. Discrete and continuous variables represent the chromosoma of individual in the population. The following example illustrates the codification of a given individual:

\begin{tabular}{cc|c|c|} 
& \multicolumn{1}{c}{$\begin{array}{c}\text { Fault location } \\
(\mathrm{km})\end{array}$} & Fault type & \multicolumn{1}{c}{$\begin{array}{c}\text { Fault } \\
\text { Impedance }(\Omega)\end{array}$} \\
\cline { 2 - 4 } Individual i & 325.34 & $\begin{array}{c}\text { Phase-to- } \\
\text { ground }\end{array}$ & 12.34 \\
\hline
\end{tabular}

Fig. 1. Individual codification

Fault site: this gene represents a continuous variable that gives a possible point where the fault occurred. All transmission lines are grouped to form a chain with total length equal to the sum of the lengths of each line in the power network.

Fault type: this gene represents a discrete variable that gives the possible fault type that occurred in that specific site. Four different types of fault were considered, namely, three-phase, double-phase, phase-to-ground and double-phase-to-ground short circuits.

Fault impedances: this gene represents a continuous variable related to the impedance involved in the fault, which might range from 0 to a maximum value, e.g. $20 \Omega$.

\section{Population}

The number of individuals in a population is set in advance. As for the case study, a population of 80 individuals showed to be adequate.

Failure rates in each system branch provide information for the selection of individuals for future generations. The selection was based on the "wheel-of-fortune" method [5]. Each individual in this method occupies a range in the wheel, which is proportional to its fitness function to the problem.
Regarding the short circuit types, a similar method was applied based on the probability related to each fault type, as illustrated as follows:

- Three phase fault $\quad 5 \%$

- Double-phase fault $10 \%$

- Single-phase-to-ground fault $70 \%$

- Double-phase to ground fault $15 \%$

\section{Objective Function}

The fitness function is related to the problem optimization, i.e. it bases on the objective function that provides the least squared difference between measured and calculated values:

$$
\left.f_{\text {fitness }}=\sum\left(V_{m A}-V_{c A}\right)^{2}+\left(V_{m B}-V_{c B}\right)^{2}+\left(V_{m C}-V_{c C}\right)^{2}\right]
$$

where:

$V_{m_{i} A}:$ Voltage magnitude in meter $m_{i}$, phase A;

$V_{c A}$ : Calculated voltage magnitude in phase A;

Most optimization problems are constrained. As for the proposed problem, there are no constraints, but one could include some information regarding the utility data base, or switching status provided by the SCADA system that narrows the solution space to some specific transmission lines in the power system. The calculated voltage magnitudes in equation (1) are determined by a short circuit software, named SINAP $t \& d$, that gives the voltages and currents throughout the power system, for a given fault location, fault type and fault impedance, which are the basic variables pointed out by the individual's codification.

\section{E. Selection}

There are various ways to select individuals for the following generation. In this paper, the fittest individuals are more likely to be selected to future generations. The wheel of fortune method was chosen [5], in which the probability of selecting an individual to be copied to the next generation relies on how well fit it is, i.e. the selection probability is proportional to its corresponding fitness function. 


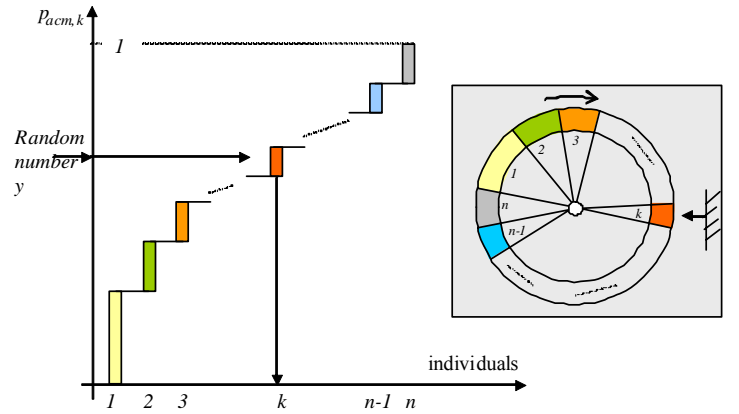

Fig. 2. Selection of an individual - wheel of fortune method

The vertical axis in figure 2 shows the normalized values of accumulated fitness functions. The accumulated probability of a given individual $\mathrm{k}$ in the horizontal axis, is given by the following expression:

$$
P_{\text {acum }, k}=\frac{\sum_{j=1}^{k} f_{\text {aval }, j}}{\sum_{j=1}^{n} f_{\text {aval }, j}}
$$

By randomly generating a number $y$ in the interval $[0,1]$, one ensures that the fittest individuals have higher probability to be selected to further generations.

\section{F. Mutation}

Mutation consists in the main evolutionary operator in ESs. This operator acts in each individual with a given rate, named mutation rate $p_{m}$, which varies in the range from 0 to 1 . The mutation is carried out provided that a random generated number in the interval $[0,1]$ is not greater that the mutation rate $p_{m}$. By considering a random Gaussian variable (mean 0 and standard deviation $\sigma$ ), the mutation is applied to a given individual $x_{i}$ by the following expression:

$$
x_{i}^{\prime}=x_{i}+\sigma \cdot N_{i}(0,1)
$$

Where $N_{i}(0,1)$ is a random number with mean equal to 0 and standard deviation equal to 1 . The standard deviation $\sigma$ determines the mutation step for the generation of descendent individuals. Such a mutation step is not a constant, that is, this is a parameter to be dynamically controlled. In reference [6], a self-adaptation technique is shown that varies the mutation step and individuals according to the following expressions:

$\sigma_{i}^{\prime}=\sigma_{i} \cdot \exp \left(\tau^{\prime} \cdot N(0,1)+\tau \cdot N_{i}(0,1)\right)$

$x_{i}^{\prime}=x_{i}+\sigma_{i}^{\prime} \cdot N_{i}(0,1)$

where:

$\sigma$ 'i: $\quad$ variation of the parameter $\sigma$ index $\mathrm{i}$; бi: $\quad$ mutation step;

$\mathrm{Ni}(0,1)$ : randomly generated number, with Gaussing distribution, mean equal to 0 and standard deviation equal to 1 ;

$\mathrm{N}(0,1)$ : randomly generated number, with Gaussian distribution, mean equal to 0 and standard deviation equal to 1 . This value is kept constant for each individual.

$$
\begin{array}{ll}
\tau, & \text { learning rate }\left(\propto(\sqrt{2 \beta})^{-1}\right) \\
\tau . & \text { learning rate }\left(\propto(\sqrt{2 \sqrt{\beta}})^{-1}\right)
\end{array}
$$

According to reference [6], the parameter $\beta$ is usually set to 2 .

Mutation of the fault site and fault impedances: In order to consider the actual geometrical distances between two possible transmission lines as well as fault impedances, an ordered list of elements was created, based on the fault site and relation of positive to zero sequence impedances, represented by the following expressions:

Single phase to ground faults:

$$
Z_{\text {equi }}=\frac{1}{2 Z_{1}+Z_{0}}
$$

Other fault types:

$$
Z_{\text {equi }}=\frac{1}{Z_{1}}
$$

Mutation of the fault type: as for the fault type, the mutation operator bases upon probability criteria, as previously described for the selection of individuals in the population.

\section{G. Crossover}

The crossover operator aims at introducing variability to the random search in the problem space. This is based on the exchange of information between individuals of the same species. One can even avoid convergence to local optima by using such evolutionary operator.

One of the ways to carry out the crossover operator consists in obtaining the parameters of a pair of individuals that have been selected or mutated by the aforementioned selection and mutation operators. This exchange of information is herein carried out by the average of the parents' characteristics, given that a randomly generated number is equal to or not greater than the given crossover rate $p_{c}$.

When the crossover is carried out, the genes to have information exchanged are randomly established. As for the fault site and fault impedance, the following expression of the pair (indiv ${ }_{1}$, indiv $\left.{ }_{2}\right)$ generates a 
descendent $\operatorname{indiv}_{3}$ :

$$
\begin{aligned}
& L_{\text {fault }}\left(\text { indiv }_{3}\right)=\frac{L_{\text {fault }}\left(\text { indi }_{1}\right)+L_{\text {fault }}\left(\text { indiv }_{2}\right)}{2} \\
& \left.Z_{\text {fault }\left(\text { indiv }_{3}\right)}\right)=\frac{\left.Z_{\text {fault }\left(\text { indiv }_{1}\right)}\right)+Z_{\text {fault }\left(\text { indiv }_{2}\right)}}{2}
\end{aligned}
$$

Where $L_{\text {fault }}$ and $Z_{\text {fault }}$ represent the fault site and the fault impedance, respectively.

Fig. 2 illustrates the overall methodology based on Evolutionary Strategies.

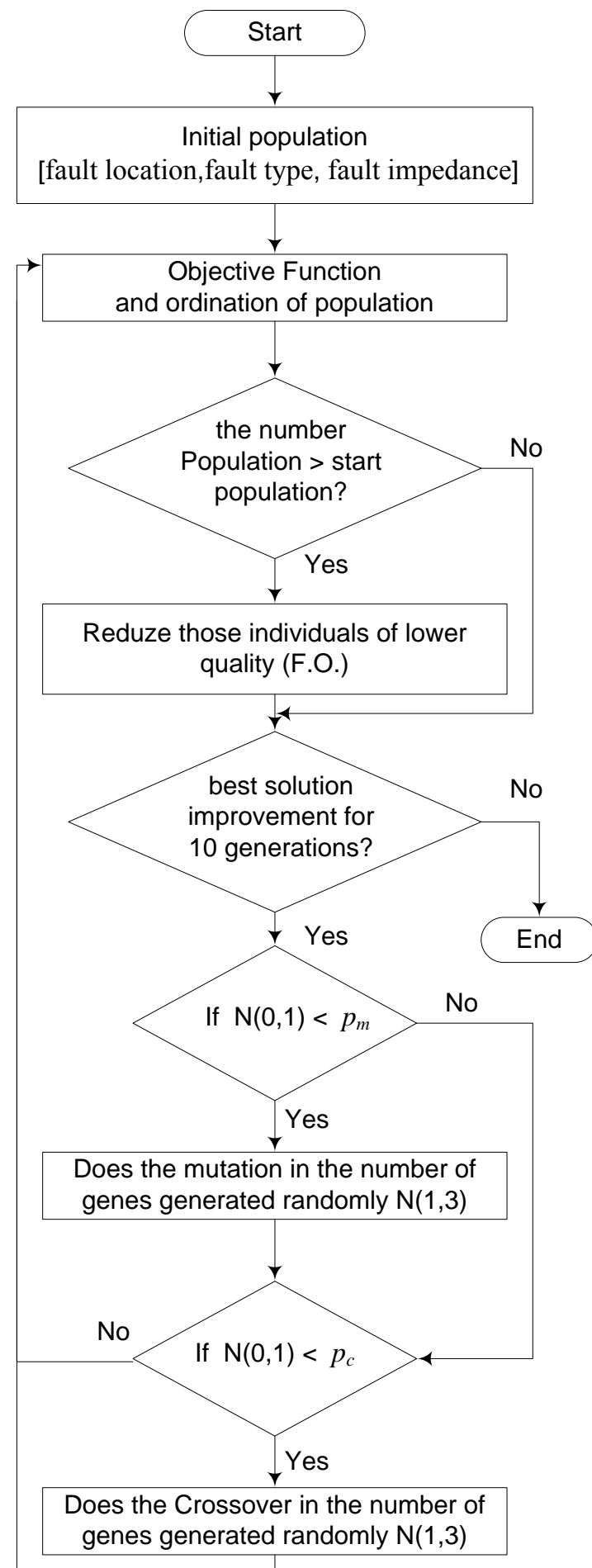

Fig. 3. Fault location flowchart

\section{Case Study}

This section shows the application of the proposed method to a 30 bus IEEE test network. The method was implemented in a $\mathrm{C}++$ programming language environment, which composes the developing power system package named SINAP.

The network is illustrated in Fig.3. The total length of the network is $360 \mathrm{~km}$, which encompasses three voltage levels, namely $132 \mathrm{kV}, 33 \mathrm{kV}$ and $11 \mathrm{kV}$. Three power quality meters were randomly allocated in system buses to register voltage magnitudes, which are triggered by the occurrence of voltage sags (magnitude less than or equal to $0,9 \mathrm{pu})$.

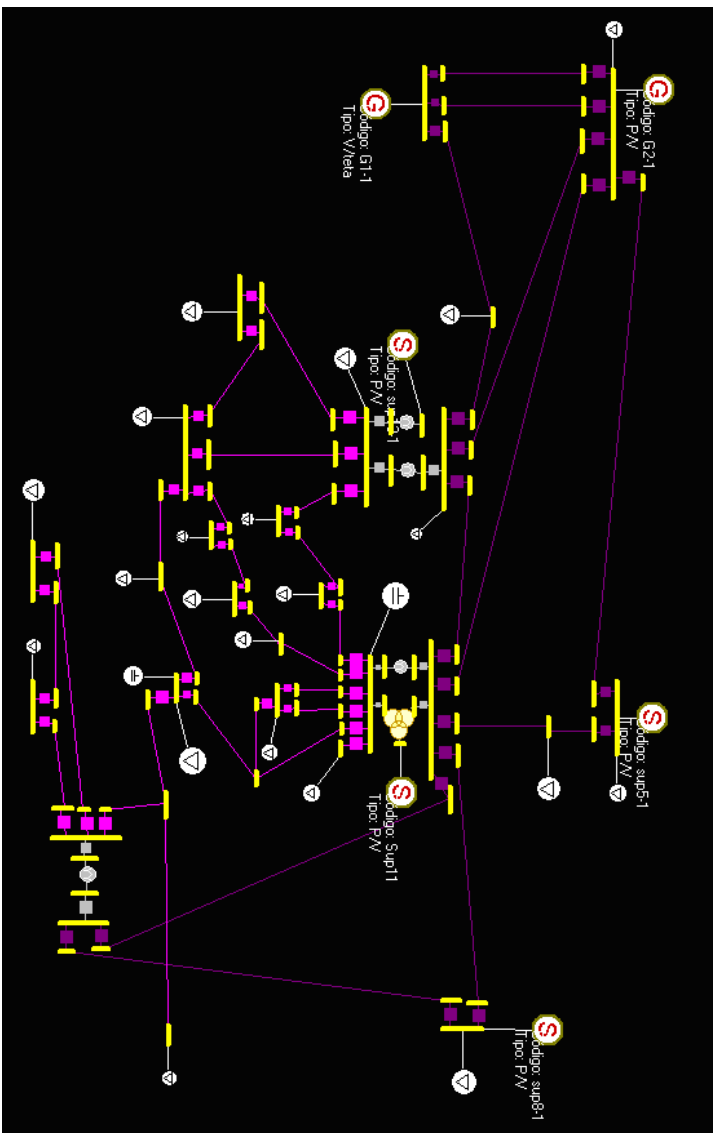

Fig. 3. 30 Bus IEEE network

Table 1 shows results for three events which were simulated by using the methodology. One can notice that the errors in fault location are inferior to $0,5 \mathrm{~km}$, what shows the potentiality of the proposed method. 
Table 1 - Results for the 30 bus IEEE network

\begin{tabular}{|c|c|c|c|c|}
\hline $\begin{array}{c}\text { Network } \\
\text { branch }\end{array}$ & $\begin{array}{l}\text { Fault site } \\
\text { (distance } \\
\text { in } \mathrm{km} \text { ) }\end{array}$ & Fault type & $\begin{array}{c}\text { Fault } \\
\text { impedance } \\
(\Omega)\end{array}$ & $\begin{array}{l}\text { Fitness } \\
\text { function }\end{array}$ \\
\hline LT628-1 & 6.75 & Phase-ground & 11.25 & $\begin{array}{c}\text { Fault site } \\
\# 1\end{array}$ \\
\hline LT628-1 & 7.00 & Phase-ground & 11.16 & $2.19 \mathrm{E}-6$ \\
\hline LST1214-0 & 3.46 & Phase-ground & 15.30 & $\begin{array}{c}\text { Fault site } \\
\# 2\end{array}$ \\
\hline LST1214-1 & 3.50 & Phase-ground & 16.46 & $4.35 \mathrm{E}-6$ \\
\hline LST1215-1 & 7.25 & Three-phase & 0 & $\begin{array}{c}\text { Fault site } \\
\# 3\end{array}$ \\
\hline LST1215-1 & 6.00 & Three-phase & 0 & $2.63 \mathrm{E}-5$ \\
\hline $\begin{array}{r}\text { * lines in } \\
\text { lines }\end{array}$ & repres & $\begin{array}{l}\text { e given fau } \\
\text { sent the obt }\end{array}$ & $\begin{array}{l}\text { and impe } \\
\text { ite and } i\end{array}$ & $\begin{array}{l}\text { e whereas } \\
\text { lance }\end{array}$ \\
\hline
\end{tabular}

The method's performance is obviously related to the number of power quality meters as well as their allocation in the system so that they can "observe" faults for any (or most of ) fault type, location and impedance.

\section{Conclusions}

This paper has presented the development of a new method to fault location in transmission lines, by using Evolutionary Strategies. The method was successfully applied to the 30 bus IEEE test system.

The proposed evolutionary operators, regarding selection, mutation and crossover are proven adequate, since fault location errors are not greater than $2 \%$.

The creating of an ordered list of fault sites with respective equivalent sequential impedances (according to expressions 6 and 7) did help to direct the search so that the mutation operator could behave in a much better way.

The self-adaptation strategy utilized for the mutation process has made the algorithm to automatically control the mutation steps in a way to intensify and diversify the search in the solution space.

An important aspect relates to the power quality meters' "observability". This concept is related to how a given network fault triggers the installed power quality meters. The power quality meters are not installed in the network to optimize fault location algorithms. An optimal allocation of power quality meters can help the propose algorithm so that the fault sites and impedances tend to be much more accurate.

\section{Acknowledgement}

The authors wish to thank CAPES - Brazilian Research Foundation for the support during the development of this work.

\section{References}

[1] Mora. J. "Voltage Sag Characterization and Classification for Diagnosis in Electric Power Quality Domain", Master dissertation. University of Girona, España. 2003

[2] Calhau D. Vinicius D. André C. and Carvalho A. "Localização de Faltas em Linhas de Transmissão de Energia Elétrica Utilizando Reconhecimento de Padrões", Proceedings of the IV Brazilian Conference on Neural Networks - IV Congresso Brasileiro de Redes Neurais, pp 061-066, 1999.

[3] Steinder M. and Sethi S. "Probabilistic Fault Localization in Communication Systems Using Belief Networks", IEEE/ACM Transactions on Networking, Vol. 12, No. 5, October 2004

[4] D. E. Goldberg: Genetic Algorithms in Search, Optimization, and Machine Learning, AddisonWesley Publishing Company, Inc. USA, 1953.

[5] N. Kagan: Configuração de Redes de Distribuição Através de Algoritmos Genéticos e Tomada de Decisão Fuzzy, Tese de Livre Docência: EPUSP, 1999.

[6] T. Back and H.-P. Schwefel, "Evolutionary Computation: An Overview," in Proceedings of IEEE International Conference on Evolutionary Computation, 1996, pp. 20-29. 